\title{
Tonsillar metastasis of gastric cancer
}

\author{
Emi Yamaguchi $\cdot$ Masaaki Uchida $\cdot$ Yoshinari Makino $\cdot$ \\ Maromi Tachibana - Takashi Sato - Yoshio Yamamoto • \\ Kousaku Kawashima $\cdot$ Asuka Araki $\cdot$ Riruke Maruyama
}

Received: 19 January 2010/Accepted: 17 August 2010/Published online: 17 September 2010

(C) Springer 2010

\begin{abstract}
Metastasis from a malignant tumor to the palatine tonsils is rare, with only 100 cases reported in the English-language literature. Tonsillar metastasis from a gastric cancer is very rare. We report here a case of palatine tonsillar metastasis after gastric cancer surgery. The patient was an 88-year-old woman who had gastric cancer with abdominal wall invasion. She had undergone a distal gastrectomy with abdominal wall resection and D2 lymph node dissection. Histologically, the tumor was primarily a moderately differentiated adenocarcinoma. It was stage IV (T4, N1, M0) using TNM clinical classification. The patient developed pharyngeal discomfort and abdominal pain and was hospitalized during the follow-up period, 1 year 9 months post-operatively. Multiple lung metastases, Virchow's lymph node metastasis, and adrenal metastasis were observed. A mass of $2.5 \mathrm{~cm}$ was also observed in the right palatine tonsil. It was diagnosed as a moderately differentiated adenocarcinoma, a metastasis from gastric cancer. There was a concern of asphyxiation due to hemorrhage of the tumor; however, the tumor dislodged approximately 10 days after biopsy and tonsillar recurrence was not observed. The patient died 1 year 10 months post-operatively. In the literature there are cases
\end{abstract}

E. Yamaguchi $(\bowtie) \cdot$ M. Uchida $\cdot$ Y. Makino - M. Tachibana

T. Sato · Y. Yamamoto

Department of Surgery, Matsue Seikyo Hospital,

8-8-8 Nishitsuda, Matsue, Shimane 690-8552, Japan

e-mail: emi-med-ml@poppy.ocn.ne.jp

K. Kawashima

Department of Internal Medicine, Matsue Seikyo Hospital,

Matsue, Japan

A. Araki · R. Maruyama

Organ Pathology Unit, Department of Pathology,

Shimane University Faculty of Medicine, Shimane, Japan with tonsillar metastases where surgical treatment, radiotherapy, and chemotherapy were performed and extension of survival was seen. Tonsillar metastasis is a form of systemic metastasis of a malignant tumor, and there is a high risk for asphyxiation from tumor dislodgement or hemorrhage. Thus, it is important to recognize tonsillar metastasis as an oncologic emergency.

Keywords Tonsillar metastasis - Gastric cancer . Oncologic emergency

\section{Introduction}

Malignant tumors that develop in the palatine tonsils are mostly primary malignancies. There have only been 100 cases of metastatic tumors involving the tonsils reported in the English-language literature [1]. Tonsillar metastasis from a gastric cancer is very rare.

We report here a case with tonsillar metastasis 1 year 9 months after gastric cancer surgery and include a brief literature review.

\section{Case report}

The patient was an 88-year-old woman who had undergone gastric cancer surgery and was seen at the outpatient clinic. She developed anemia at age 87 and was diagnosed with gastric cancer by an upper gastrointestinal endoscopy. Gastric cancer was Borrmann type 3 occupying approximately half the circumference and located near the lesser curvature in the anterior wall of the lower gastric body. An examination showed that there was no distant metastasis. The patient was scheduled for surgery, but she developed 
myocardial infarction and the surgery was postponed. When her general condition improved after 6 months, surgery was performed. Pre-operative imaging showed an
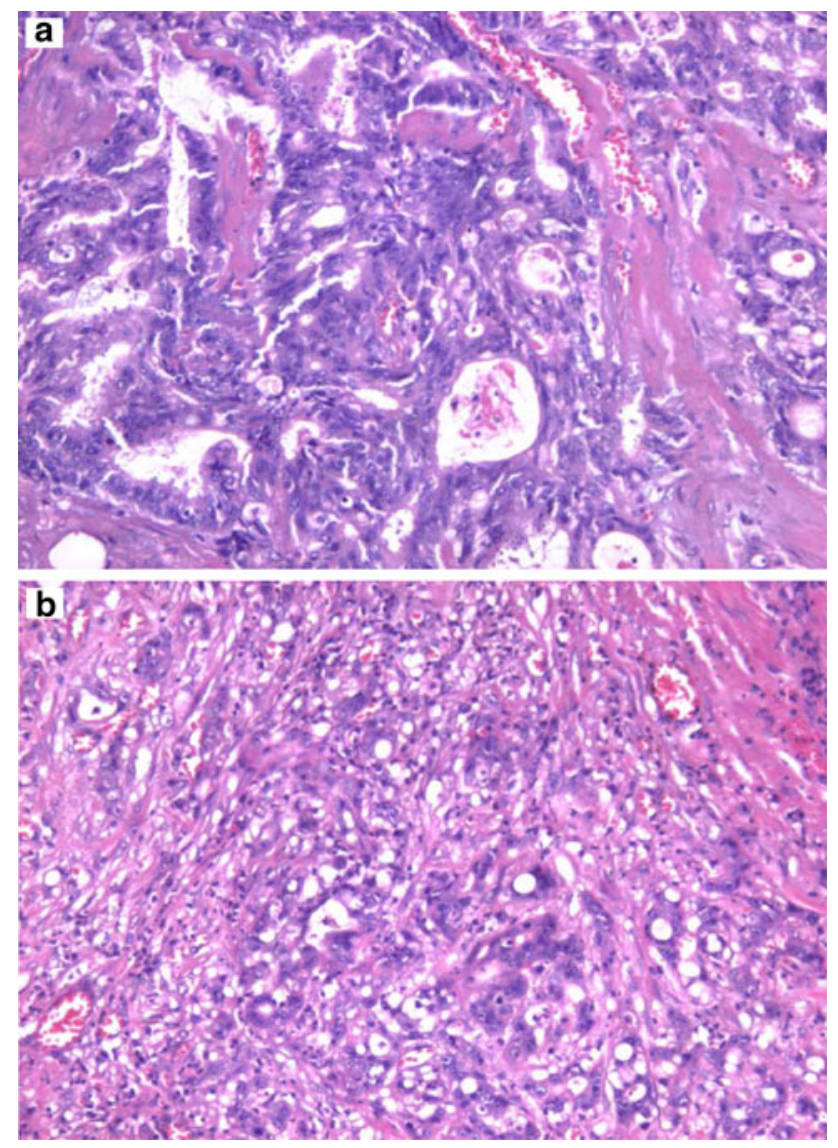

Fig. 1 Microscopic findings $(H \& E$ staining, $\times 100)$ of the resected primary gastric adenocarcinoma. a The tumor consisted of a moderately differentiated adenocarcinoma, and $\mathbf{b}$ a poorly differentiated adenocarcinoma

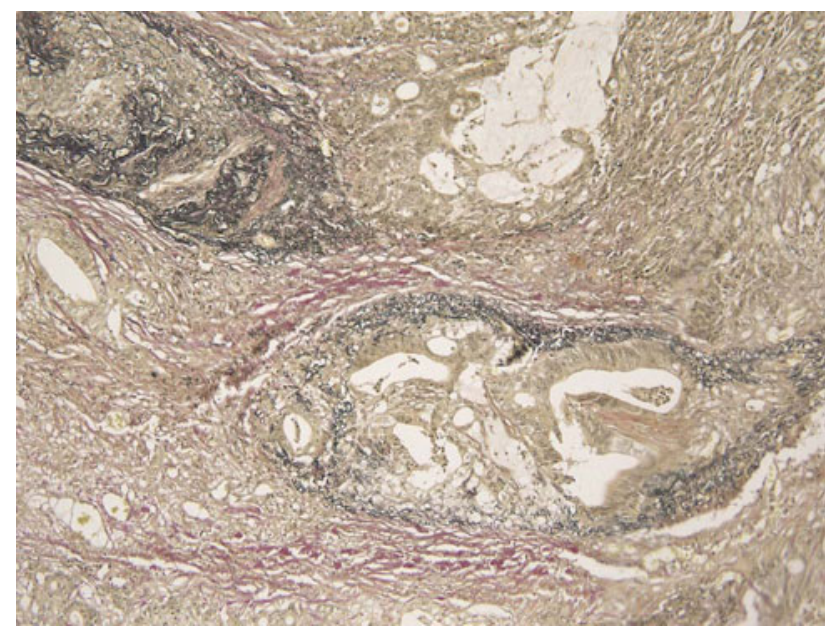

Fig. 2 Microscopic findings (Elastica van Gieson staining, $\times 100$ ) of the resected stomach. Both veins and lymphatic vessels were stained. Furthermore, there was intense staining of a very large vein abdominal wall invasion of gastric cancer but no distant metastasis. A distal gastrectomy with abdominal wall resection and a lymph node dissection were performed. Histologically, the resected specimens were determined to be gastric cancer, mainly a moderately differentiated but also partly a poorly differentiated adenocarcinoma (Fig. 1). The tumor was stage IV (T4, N1, M0) according to TNM clinical classification. Additional histologic findings were high venous invasion (V1) and middle-level lymphatic vessel invasion (L1). According to the Japanese classification of gastric carcinoma, the findings were $\mathrm{v} 3$ and ly2, respectively. We were not able to confirm venous invasion and lymphatic vessel invasion at the site of the tonsillar metastasis, because the tonsils were tissue of the biopsy. Venous and lymphatic vessel invasion was found in the stomach tissue. Furthermore, invasion of a very large vein was seen on Elastica van Gieson staining (Fig. 2). Chemotherapy was not performed post-operatively as requested by the patient's family, and the patient was followed up on an outpatient basis. Left adrenal metastasis was suspected 1 year 6 months post-operatively, but there were no
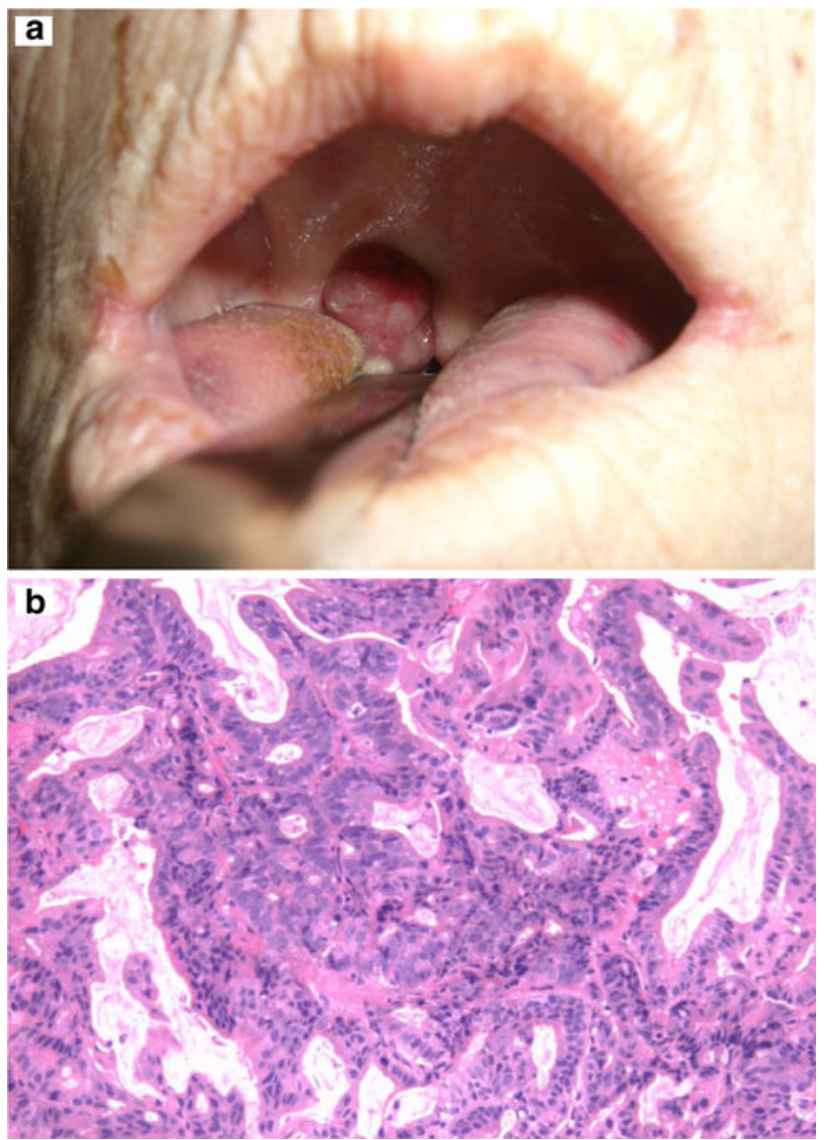

Fig. 3 Palatine tonsil metastasis. a An approximately $2.5 \mathrm{~cm}$ tumor was found at the right palatine tonsil. b Microscopic findings ( $\mathrm{H} \& \mathrm{E}$ staining, $\times 100$ ) of the biopsied samples showed a moderately differentiated adenocarcinoma 
symptoms. Since the patient requested follow-up, she continued to be monitored on an outpatient basis. She complained of pharyngeal discomfort and abdominal pain 1 year 9 months post-operatively, and she was hospitalized for further examination.

During hospitalization, a $2.5 \mathrm{~cm}$ tumorous lesion was observed in the right palatine tonsil (Fig. 3a). A subcutaneous mass was observed in the occipital region, and a Virchow's lymph node was palpable. The abdomen was flat and soft, and a mass was not palpable. A contrastenhanced thoraco-abdominal computed tomography scan showed the enlargement of Virchow's lymph node metastasis, multiple lung metastases, and left adrenal metastasis. Brain metastasis was suspected in the contrast-enhanced magnetic resonance imaging scan of the head. A palatine tonsillar mass was not observed. Neither liver metastasis nor ascites were found. Carcinoembryonic antigen (CEA) and carbohydrate antigen 19-9 (CA19-9) levels were elevated at $5.8 \mathrm{ng} / \mathrm{ml}$ and $7010 \mathrm{U} / \mathrm{ml}$, respectively.

The palatine tonsillar mass was $2.5 \mathrm{~cm}$ with a hematoma. A biopsy showed a moderately differentiated adenocarcinoma and it was diagnosed as a metastasis from the gastric cancer (Fig. 3b). The subcutaneous mass of the head was also an adenocarcinoma and a metastasis from the gastric cancer.

The patient had reduced oral food intake at admission due to pharyngeal discomfort. Since there was a risk for asphyxiation from a tonsillar tumor hemorrhage or dislodgement, further examination was performed with consideration for resection. The mass spontaneously dislodged approximately 10 days after biopsy. The subjective symptoms were also resolved and the patient was able to take food orally. Thereafter best supportive care (BSC) was continued and the patient died 1.5 months after hospitalization (1 year 10 months post-operatively).

\section{Discussion}

Malignant tumors involving tonsils are mostly primary tumors; metastatic tumors are rare. Gallo et al. [1] reported that there were approximately 100 cases published in the English-language literature. Many tonsillar metastases are from head and neck cancer [2], but there are also many metastases from renal cancer and melanoma, followed by breast cancer and lung cancer. In 1965, Friedmann et al. [3] reported on tonsillar metastasis from gastric cancers in a review of 71 cases of metastatic cancer to the head and neck. They reported that there were only two cases (tonsil and nasopharynx) of metastases from gastric cancer to the same region.

We performed English and Japanese literature searches of publications up to March 2010 on gastric cancer metastasis to the head and neck, excluding brain and cervical lymph node metastasis, using PubMed and ICHUSHI (Japan Medical Abstracts Society) digital archives. We found 33 cases with sufficient information for review [1, 4-32].

Table 1 summarizes information on these 33 cases and our own case. The age of onset ranged from 42 years to 88 years, with a mean age of 62.4 years. Our 88 -year-old patient was the oldest case.

The duration of time between the diagnosis of gastric cancer and metastasis was determined. There were 13 metachronous cases in which the interval between the diagnosis of gastric cancer and metastasis was greater than 1 year, with a mean interval of 25.2 months. There were 19 synchronous cases, in which the interval to metastasis was less than 1 year. Ten of these 19 cases were diagnosed with gastric cancer and a metastatic lesion at the same time. The mean interval in the 9 cases diagnosed with metastasis occurring longer than 1 month after the primary diagnosis was 5 months. In addition, 2 of the cases were patients in whom the metastatic lesion had already been identified before the diagnosis of gastric cancer.

There were 28 cases with a histopathological diagnosis available for review. There were $9(32.1 \%)$ well-tomoderately differentiated adenocarcinomas and 19 (67.9\%) poorly or undifferentiated adenocarcinomas or signet-ring cell carcinomas. Many gastric cancers tended toward low-grade differentiation.

The location of gastric cancer metastasis to the head and neck were reviewed in all 34 cases. There were 13 (38.2\%) with tonsillar, $8(23.5 \%)$ with thyroid, $6(17.6 \%)$ with gingival, and 4 cases $(11.8 \%)$ with dural metastases. In addition, there was 1 case each of metastasis to the pituitary gland, the submaxillary gland, and the oral mucosa.

With regard to gastric cancer tonsillar metastasis, there were 3 cases $(23.1 \%)$ with bilateral and 10 cases (76.9\%) with unilateral metastases. Six cases had unilateral metastasis on the left and 4 cases were on the right.

In general, the clinical and histopathologic diagnosis of metastatic tonsillar carcinoma is considered to be difficult; however, it should be recognized and treated as a systemic metastatic lesion. Most primary tonsillar carcinoma lesions are of the epidermoid type with various levels of differentiation; undifferentiated (anaplatic) carcinoma is rare. Although salivary gland tumors (pleomorphic adenomas and adenoid-cystic carcinomas) can occur in the tonsillar region, they rarely show characteristics of adenocarcinoma. Tonsillar adenocarcinoma is therefore highly likely to metastasize. If tonsillar adenocarcinoma is seen bilaterally, metastasis is even more likely. Physicians should be aware of this and proceed with appropriate examinations.

Of the cases we reviewed, 6 cases of tonsillar metastasis were treated. Five patients underwent surgery (1 case 


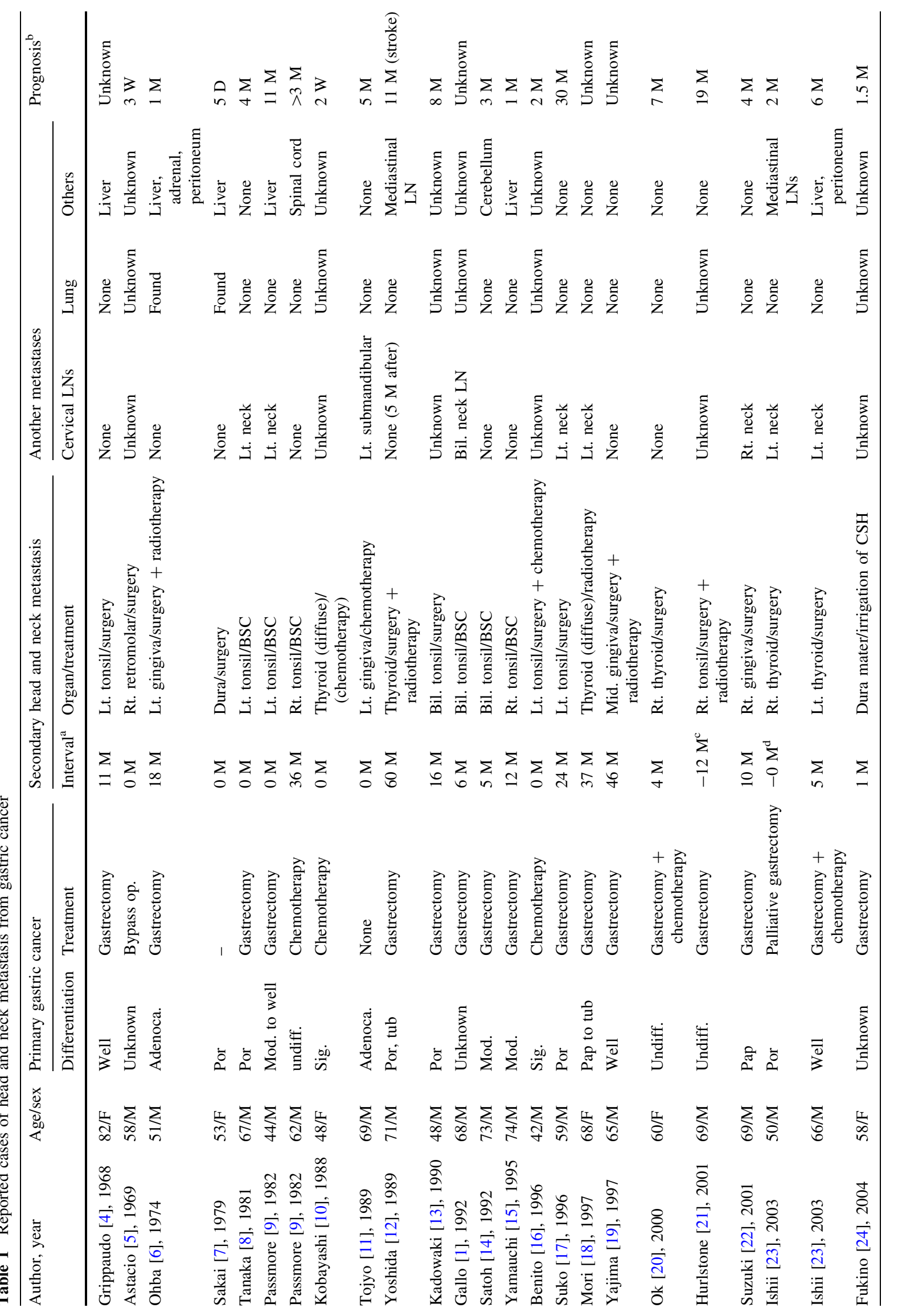




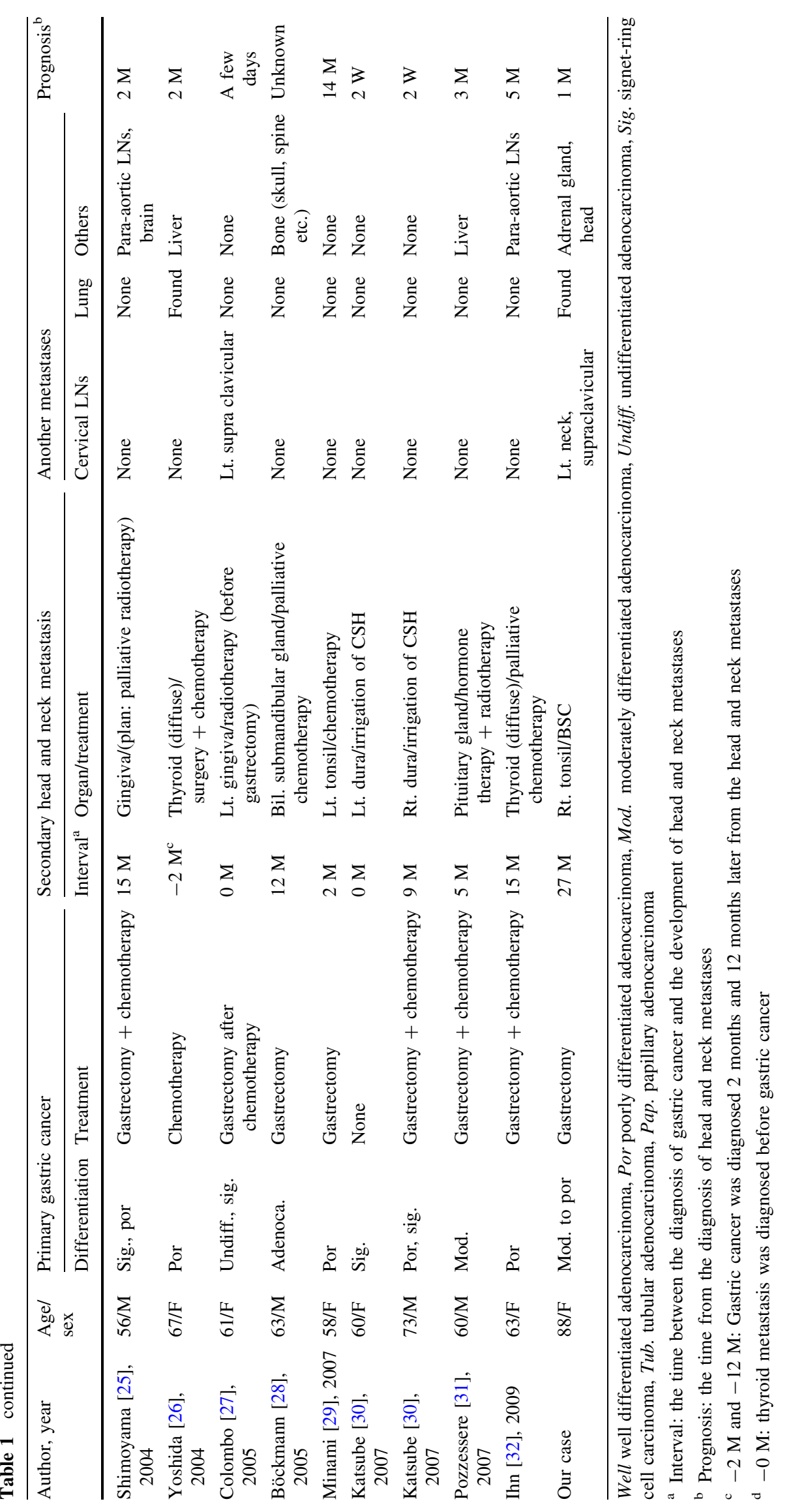


combined with chemotherapy and 1 case combined with radiotherapy) and there was 1 case treated only with chemotherapy. BSC was provided to another 7 patients.

The duration of survival was reported in 5 treated patients. The longest survival was 30 months, with a mean survival of 14.6 months. By contrast, among the patients receiving BSC, the mean survival for 6 of these patients was 3.8 months. These data indicate that surgical treatment, chemotherapy, and radiotherapy were effective in tonsillar metastasis from gastric cancer.

Tonsillar metastasis is a systemic malignant tumor metastasis. Treatment should be considered, because longterm survival may be possible. In our case, there was a risk of asphyxiation from tonsillar tumor hemorrhage or dislodgement. Therefore, the patient was evaluated for resection of the tonsillar metastasis; however, spontaneous dislodgement of the tumor occurred approximately 10 days after biopsy.

Theories on the mechanisms of metastasis to the tonsils include: (1) hematogenous metastasis, (2) lymphatic metastasis, (3) metastasis via paravertebral plexus from lung metastasis, which in turn is from head and neck cancer[33], (4) direct invasion from surrounding lymph node metastasis, and (5) direct seeding to the tonsillar crypts due to regurgitation of the gastric content [34]. Many hematogenous metastases (1) have been said to metastasize from the lungs via Batson's vertebral venous plexus [35]. A metastatic route is speculated from portal circulation through the liver or lungs to the systemic circulation via the heart and finally reaching the tonsils [35]. For lymphatic metastasis (2), retrograde metastasis could occur from the thoracic duct via the cervical lymph nodes because there are no afferent lymphatic vessels in the tonsils [36]. For direct invasion from a metastasis of a surrounding lymph node (4), the mechanism is thought to involve retrograde metastasis from the thoracic duct to a cervical lymph node; however, the majority of the cases do not have obvious metastases in the cervical lymph nodes. Therefore, for tonsillar metastasis, this mechanism is unlikely, and hematogenous metastasis (1) is generally considered most probable.

Our case had a metastasis involving the right tonsil and a cervical lymph node metastasis on the left. Multiple lung metastases were also observed, and hematogenous metastasis via the lung was highly likely. The metastasis to the tonsils was thought to be hematogenous metastasis because we recognized an invasion of a very large vein on sections stained with Elastica van Gieson stain.

Finally, in our case, the tonsillar metastatic lesion was spontaneously dislodged, and the tumor did not occlude the airway. Airway occlusion by hemorrhage or blood clot did not occur either, but there was a risk. Yamauchi reported on a case needing intubation because of bleeding from a tonsillar metastasis and dyspnea which indicates that tonsillar metastasis should also be recognized as an oncologic respiratory emergency.

\section{References}

1. Gallo A, Pescarmona E, Crupi J, Corsetti GL, Vincentiis MD. Bilateral tonsillar metastasis of gastric adenocarcinomas. Head Neck. 1992;14:55-7.

2. Golas SM. Trends in palatine tonsillar cancer incidence and mortality rates in the United States. Commun Dent Oral Epidemiol. 2007;35:98-108.

3. Friedmann I, Osborn DA. Metastatic tumours in the ear, nose and throat region. J Laryngol Otol. 1965;79:576-91.

4. Grippaudo M. A case of metastatic adenocarcinomas in the tonsil. J Laryngol Otol. 1968;82:645-8.

5. Astacio JN, Alfaro C. Oral mucosa metastasis from gastric adenocarcinoma. Oral Surg Oral Med oral Pathol. 1969;28:859-61.

6. Ohba T, Nakagawa E, Nakata H, Takeda N, Matuura K, Katayama H. Metastatic tumor of the gum. Jpn J Cancer Clin. 1974;20:692-6.

7. Sakai S, Mori Y, Matsuoka K. Metastatic dural carcinomatosis secondary to gastric cancer. Neuro Med Chir. 1979;19:39-44.

8. Tanaka N, Toya M, Koyanagi Y. Tonsillar metastasis from carcinoma of the stomach: report of a case. Otologia Fukuoka. 1981;27:811-4.

9. Passmore AL, Hugh TB, Coleman MJ. Tonsillar metastases from adenocarcinomas of the stomach. Aust N Z J Surg. 1982; $52: 371-2$.

10. Kobayashi K, Yashiro T, Suzuki A, Manabe Y, Ozaki O, Ito K. Two cases of metastatic tumor to the thyroid gland. J Jpn Surg Assoc. 1988;49:814-9.

11. Tojyo Y, Saito K, Aihara H, Tuda Y, Sisimaru Y. The metastasis of gastric adenocarcinoma to the gingival of the mandiblereview of the Japanese literature. Gan No Rinsho. 1989;35: 1165-70.

12. Yoshida A, Imamura A, Tanaka H, Hirano M, Kamma H, Ueno E, Ushio H, Aiyoshi Y, Soeda S. A case of metastasis from gastric cancer to the thyroid gland. Jpn J Surg. 1989;19:480-4.

13. Kadowaki K, Ikoma H. Metastatic carcinoma of the bilateral tonsils owing to gastric cancer: a case report. Otologia Fukuoka. 1990;36:1144-6.

14. Satoh R, Hendo M, Nagashima M. Metastatic carcinoma of bilateral palatine tonsils from the stomach. Otolaryngol Head Neck Surg. 1992;64:921-5.

15. Yamauchi H, Nishihira S, Hirano $Y$, Watanabe H. Tonsillar metastasis from gastric cancer-a case report. Otolaryngol Head Neck Surg. 1995;67:650-4.

16. Benito I, Alvarez-Gago T, Morais D. Tonsillar metastasis from adenocarcinoma of the stomach. J Laryngol Otol. 1996;110: 291-3.

17. Watanabe T, Suko T, Mogi G, Arita K. Tonsillar metastasis from gastric cancer. Stomato Pharyngol. 1996;8:391-4.

18. Mori T, Kitamura H, Arai M, Numata M, Kasuga Y. Diffuse color Doppler signals in a case of gastric cancer that metastasized to the thyroid gland. J Med Ultrasonics. 1997;24:1841-4.

19. Yajima M, Miyazak T. Metastasis of gastric adenocarcinoma to the maxillary gingival: report of a case. Jpn J Oral Maxillofac Surg. 1999;45:25-7.

20. OK E, Sözüer E. Thyroid metastasis from gastric carcinoma: report of a case. Surg Today. 2000;30:1005-7.

21. Hurlstone DP, Sanders DS, Smith A, Jones RB, Slater DN, Bardhan KD. Tonsillar metastasis: a rare presentation of gastric carcinoma. Eur J Surg Oncol. 2001;27:328-30. 
22. Suzuki Y, Nakagawa A, Tojima H, Wada Z, Hoshimoto K, Yamaguchi H, Okumura M, Kamoshida T, Takahashi A. A case of metastatic gastric carcinoma to the mandible. Otolaryngol Head Neck Surg. 2001;73:235-8.

23. Ishii M, Nishikawa R, Masuda T, Yano H, Sakakura K, Yamazoe $\mathrm{N}$. Two cases of thyroid gland metastases from primary gastric cancer. J Clin Surg. 2003;58:989-92.

24. Fukino K, Terao K, Kojima T, Adachi K, Teramoto A. Chronic subdural hematoma following dural metastasis of gastric cancer: measurement of pre- and postoperative cerebral blood flow with $N$-isopropyl-p-1[123I] isodoamphetamine. Case report. Neurol Med Chir. 2004;44:646-9.

25. Shimoyama S, Seto Y, Aoki F, Ogawa T, Toma T, Endo H, Itouji T, Kaminishi M. Gastric cancer with metastasis to the gingiva. J Gastroenterol Hepatol. 2004;19:831-5.

26. Yoshida T, Shimooki O, Baba Y, Abe T, Sugai T, Nakamura S. A case of metastasis from gastric cancer to the thyroid gland. J Jpn Surg Assoc. 2004;65:324-7.

27. Colombo P, Tondulli L, Masci G, Muzza A, Rimassa L, Petrella D, Santoro A. Oral ulcer as an exclusive sign of gastric cancer: report of a rare case. 2005; doi:10.1186/1471-2407-5-117.

28. Böckmann RA, Schulz T, Stein H, Mielke E, Dengler K, Wolff $\mathrm{KD}$, Nolte D. Bilateral synchronous submandibular lumps in a patient with gastric carcinoma. J Oral Pathol Med. 2005;34:127-8.

29. Minami K, Tsutani Y, Suzuki T, Miyahara E, Kameda A, Noso Y. A complete response in left metastatic tonsillar tumor treated with TS-1 based chemotherapy after curative resection of gastric carcinoma: a case report. Jpn J Gastroenterol Surg. 2007;40: 1775-80.

30. Katsube T, Kikuchi T, Konnno S, Murayama M, Kobayashi R, Kuhara K, Yoshimatsu K, Shiozawa S, Shimakawa T, Naritaka Y, Ogawa K, Hagiwara S, Aiba M. Subdural hematoma associated with dural metastasis of gastric carcinoma: report of two cases. Anticancer Res. 2007;27:4339-44.

31. Pozzessere D, Zafarana E, Buccoliero AM, Pratesi C, Fargnoli R, Leo AD. Gastric cancer metastatic to the pituitary gland: a case report. Tumori. 2007;93:217-9.

32. Ihn MH, Kim YJ, Kim JJ, Cho JY, Jin SY. A case of thyroid metastasis originating from early gastric cancer. J Korean Med Sci. 2009;24:1230-3.

33. Batson OV. The role of the vertebral veins in metastatic processes. Ann Intern Med. 1942;26:38-45.

34. Kleinschmidt HJ. Tonsillar metastases of primary bronchial and gastric carcinomas. Z Laryngol Rhinol Otol. 1966;45:389-94.

35. Asami K, Yokoi H, Hattori T, Rao AJ, Yanagita N. Metastatic gall bladder carcinoma of the palatine tonsil. J Laryngol Otol. 1989;103:211-3.

36. Brownson RJ, Jaques WE, LaMonte SE, Zollinger WK. Hypernephroma metastatic to the palatine tonsils. Ann Otol Rhinol Laryngol. 1979;88:235-40. 